\title{
Hermansky-Pudlak syndrome with pulmonary fibrosis
}

INSERM

\section{Source}

INSERM. (1999). Orphanet: an online rare disease and orphan drug data base.

Hermansky-Pudlak syndrome with pulmonary fibrosis. ORPHA:231500

Hermansky-Pudlak syndrome with pulmonary fibrosis as a complication includes two types (HPS-1 and HPS-4) of Hermansky-Pudlak syndrome (HPS; see this term), a multisystem disorder characterized by oculocutaneous albinism, bleeding diathesis and, in some cases, pulmonary fibrosis or granulomatous colitis. 